\title{
A Comparative Analysis of the Efficacy and Safety of fixed Combinations of Latanoprost/Timolol vs Dorzolamide/Timolol in Primary Open Angle Glaucoma or Ocular Hypertension
}

\author{
Sukhsagar Ratol, Rani Walia, Mridu Chaudhry
}

\begin{abstract}
Background: Glaucoma is a leading cause of irreversible blindness. The fundamental problem in medical management of glaucoma is of patient compliance. An ideal drug or a drug combination is needed to slow the progression of this majorly symptomless disease.
\end{abstract}

Aim: To compare the efficacy and tolerability of the fixed combination latanoprost and timolol instilled once daily in the evening $v s$ fixed combination of dorzolamide and timolol instilled twice daily in primary open angle glaucoma or ocular hypertension.

Materials and methods: A 12-week, randomized, open, parallel group study including 50 patients with primary open angle glaucoma or ocular hypertension was conducted at a tertiary care hospital. Patients were randomized to group 1, (fixed combination (FC) latanoprost and timolol eye drops, once daily in evening) and group 2, (FC dorzolamide and timolol eye drops, twice daily). At baseline, 2, 4 and 12 weeks, IOP was recorded at $9 \mathrm{AM}$ and 12 noon. The difference in IOP reduction in two treatment groups from baseline to 12 weeks was the main outcome measure.

Results: Mean diurnal IOP was similar at baseline for both groups. Mean reduction in IOP from baseline to 12 weeks was $9.92 \mathrm{~mm} \mathrm{Hg}(p=0.001)$ in group 1 and $9.22(p=0.001)$ in group 2. The reduction in IOP in both groups 1 and 2 was statistically significant at all time intervals. There was a statistically significant advantage for group 1 at 12 weeks for both time readings ( $p=0.013$ and 0.002 respectively) as compared to group 2.

Conclusion: The fixed combination of latanoprost and timolol was more effective than that of dorzolamide and timolol in reducing mean diurnal IOP and both treatments were well tolerated. To confirm further such studies are required.

Keywords: Glaucoma, Fixed combination, Latanoprost/Timolol, Dorzolamide/Timolol.

How to cite this article: Ratol S, Walia R, Chaudhry M. A Comparative Analysis of the Efficacy and Safety of fixed Combinations of Latanoprost/Timolol vs Dorzolamide/Timolol in Primary Open Angle Glaucoma or Ocular Hypertension. J Postgrad Med Edu Res 2012;46(4):172-176.

Source of support: Nil

Conflict of interest: None declared

\section{INTRODUCTION}

Glaucoma refers to a heterogeneous group of ocular disorders that affect the optic nerve and involves a loss of retinal ganglion cells in a characteristic pattern, usually with correlating visual field defects. The common denominator of glaucoma is a characteristic optic neuropathy, which derives from various risk factors, including increased Intraocular pressure (IOP). ${ }^{1}$

Glaucoma is a leading cause of irreversible blindness. ${ }^{2}$ Statistics gathered by WHO in 2002, and published, ${ }^{3}$ show that glaucoma is the second leading cause of blindness globally, after cataracts. Data from population-based surveys indicate that one in 40 adults older than 40 years has glaucoma with loss of visual function, which equates to 60 million people worldwide being affected and 8 to 14 million being bilaterally blind. ${ }^{4}$ Even in developed countries, half of glaucoma cases are undiagnosed. In India it is estimated that there are approximately 11.2 million persons aged 40 years and older with glaucoma. Primary open angle glaucoma (POAG) is estimated to affect 6.48 million persons. ${ }^{5}$

Elevated IOP is the most important causative risk factor for development and progression of POAG and it is the only treatable risk factor for open angle glaucoma. ${ }^{6}$

A current treatment strategy is to set a target pressure for the patient to achieve, the target pressure chosen can vary based on patients age, race and disease progression, status of the optic nerve head, central corneal thickness, vascular factors but it should be less than IOP at which optic nerve damage and visual loss occurs. It is conventional practice to lower the IOP by at least one-third once glaucomatous optic neuropathy is detected. ${ }^{7}$

The therapeutic arsenal available has recently been extended with the addition of new ocular hypotensive agents like prostaglandin derivatives, local CAIs and alpha-2 adrenergic agonists. ${ }^{8}$

Both timolol and latanoprost are being widely used as first line drugs in glaucoma, due to their high efficacy and limited side effect profile. Dorzolamide is another potent drug used in the treatment of glaucoma as it not only decreases the IOP but also improves ocular perfusion. In spite of having good potency, these drugs are sometimes unable to lower the IOP when used as a monotherapy and adding another drug to the therapy is required. However, 
the fundamental problem in medical management of glaucoma is of patient compliance. Prescribing a fixed combination (FC) formulation for these patients generally is deemed preferable to multidrug regimes. In terms of optimizing IOP control, minimizing visual field deterioration, preventing visual disability and minimizing associated healthcare costs, FCs are an important component of glaucoma management. ${ }^{9,10}$ The combination of latanoprost and timolol is shown to have an additive IOP lowering effect. ${ }^{11,12}$ Another available effective FC product combines timolol $0.05 \%$ and dorzolamide $2.0 \%$, a topical carbonic anhydrase inhibitor which acts similarly to timolol in suppressing aqueous humor production ${ }^{13}$ but is dosed twice daily.

The purpose of this study was to compare IOP reductions after treatment with FC latanoprost/timolol administered OD in the evening with reductions after twice daily administration of FC dorzolamide/Timolol over a 12 weeks period

\section{MATERIALS AND METHODS}

\section{Study Design}

A randomized 12-week, parallel group, open study was conducted in Department of Pharmacology in association with Department of Ophthalmology at MMIMSR, Mullana.

The study protocol was approved before the commencement of the study by the institutional ethics committee. The study was in accordance with standards set in declaration of Helsinki of 1975. All the patients gave their consent before enrolment in the study. As patients enrolled in the trial, they were randomized according to a computer generated randomization schedule. The drop-outs were not taken into consideration as it was an intention-totreat trial.

50 POAG patients, fulfilling all of inclusion criteria and exclusion criteria were selected and one eye of bilateral POAG cases was taken up as study eye.

Inclusion criteria were a male or female patient with a minimum age 25 years having a history of unilateral or bilateral primary open angle glaucoma or ocular hypertension with IOP > $22 \mathrm{~mm} \mathrm{Hg}$, diagnosed by applanation tonometry and gonioscopy, requiring pharmacological therapy, showing inadequate response to monotherapy (target IOP not achieved) and having corrected visual acuity 20/40 or greater. Gonioscopy was done to diagnose open angle glaucoma.

Exclusion criteria included the patient being diagnosed as closed anterior chamber angle, ocular injury, ocular surgery within previous 6 months, allergy to study drugs, ocular infection and other systemic illnesses.
Washout period was given to patients and varied from 1 week for patients on cholinergic agonists and CA inhibitors to 2 weeks for patients on adrenergic receptor agonists to 4 weeks for patients on beta adrenergic antagonists or prostaglandins. Patients returned weekly for IOP monitoring during this period.

Study visits included day 0 (baseline visit), 2, 4 and 12 weeks. Prestudy examination (an investigation for baseline data) included medical history, ocular history, treatment history, complete ocular examination including visual acuity, intraocular pressure, oblique examination, slitlamp examination, gonisocopy, visual fields and systemic examination.

After attaining the baseline data patients were put on treatment accordingly.

\section{Study Drugs}

- Latim eye drops (Fixed combination of latanopost $0.05 \%$ and timolol $0.5 \%$ ) once a day in the evening.

- Dorzox T eye drops (Fixed combination of dorzolamide $2 \%$ and timolol $0.5 \%$ ) twice daily morning and evening. Group 1 included patients diagnosed as primary open angle glaucoma/ocular hypertension with IOP > $22 \mathrm{~mm} \mathrm{Hg}$ and were instilled fixed combination of latanoprost $0.05 \%$ and timolol $0.5 \%$ once a day in the evening for 12 weeks.

Group 2 included patients diagnosed as primary open angle glaucoma/ocular hypertension with IOP > 22 mm Hg instilled fixed combination of dorzolamide $2 \%$ and timolol ophthalmic solution $0.5 \%$ twice daily for 12 weeks.

IOP observations were made at, 2nd, 4th and 12th week by the same investigator each time. IOP was taken at both 9 AM and 12 noon. Other parameters recorded on subsequent visits: Visual acuity, cup to disk ratio, visual fields, conjunctival hyperemia, blood pressure, pulse rate.

At each visit, the patients were asked about any adverse drug reactions. Any adverse events reported by the patient or investigator were recorded and analyzed. Safety/tolerability evaluation was based upon self-reported adverse effects.

The primary end-point included reduction in IOP from baseline to week 12. Data were collected and the analysis of each variable was performed on the change from baseline values using two tailed Student t-test. A p-value less than or equal to 0.05 was considered statistically significant. In addition, the demographic variables were analyzed using Pearson's Chi-square test.

\section{RESULTS}

Fifty patients were randomized to group 1, who instilled fixed combination of 0.005 latanoprost and $0.5 \%$ timolol once daily and group 2 in whom $2 \%$ dorzolamide + timolol $0.5 \%$ twice daily was prescribed. 
Treatment groups were statistically similar at baseline. In group 1 the age of patients ranged from 40 to 70 years with the mean age of $55.28 \pm 8.87$ years. In group 2 the age of patients ranged from 42 to 80 years with the mean age of $56.60 \pm 10.51$ years.

There was no statistically significant difference between the ages of the patients of the two groups $(p=0.6)$. The difference in male female ratio was also not statistically significant between the two groups ( $p=0.382)$ (Table 1).

In group 1 at $9.00 \mathrm{AM}$ the mean IOP decreased from $27.88 \pm 2.18$ to $17.92 \pm 0.86 \mathrm{~mm} \mathrm{Hg}$, thus resulting in a fall of $9.96 \mathrm{~mm} \mathrm{Hg}$ (35.82\%). Whereas at $12 \mathrm{am}$ the mean IOP decreased from $27.72 \pm 2.30$ to $17.84 \pm 0.688 \mathrm{~mm} \mathrm{Hg}$ resulting in a fall of $9.88 \mathrm{~mm} \mathrm{Hg}$ (35.53\%). The fall of IOP was statistically significant at all time intervals on all visits.

In-group 2 at 9 AM the mean IOP decreased from $27.56 \pm 2.10$ to $18.52 \pm 0.770 \mathrm{~mm} \mathrm{Hg}$ which amounts to fall of $9.04 \mathrm{~mm} \mathrm{Hg}$ (32.51\%). Whereas at $12 \mathrm{AM}$ the mean IOP was reduced from the baseline of $27.92 \pm$ 2.43 to $18.52 \pm 0.770 \mathrm{~mm} \mathrm{Hg}$ thus, resulting in a fall of $9.40 \mathrm{~mm} \mathrm{Hg}$ (33.81\%) (Graphs 1 and 2). The fall of IOP was statistically significant at all time intervals.

The difference in final IOP achieved in the two groups was not statistically significant for 2 and 4 weeks periods but at week 12 the latanoprost/timolol combination showed more efficacy. The decrease in mean diunal IOP from baseline to 12 weeks in group 1 was $9.920 \pm 2.519$. In group 2 this reduction was $9.220 \pm 2.25$. The difference in IOP reduction between two groups was statistically significant at 12 weeks $(p=0.004)$ (Table 2).

The mean CD ratio in group 1 increased from mean of $0.592 \pm 0.1498$. to $0.600 \pm 0.1581$. In group 2 , the mean CD ratio increased from baseline of $0.624 \pm 0.1422$ to $0.632 \pm 0.1520$. However, the increase of $\mathrm{CD}$ ratio in both groups was statistically not significant $(\mathrm{p}=0.161)$.

The mean visual acuity in group 1 decreased from $1.304 \pm 2.652$ to $1.296 \pm 2.655$ and in group 2, it decreased from $0.740 \pm 0.200$ to $0.716 \pm 0.2304$. However, the decrease in visual acuity was statistically not significant in both the groups ( $p=0.327,0.185$ respectively).

In general both the fixed combinations were well tolerated although more patients treated with dorzolamide/ timolol FC reported eye pain after ocular instillation. Latanoprost/timolol was associated with more frequent conjunctival hyperemia. There was no significant differences in drug related ocular or systemic side effects, both the FCs were well tolerated (Table 3).

\section{DISCUSSION}

IOP is the only modifiable risk factor in POAG and medical therapy is the first therapeutic approach. In all patients IOP may not be controlled with monotherapy and adjunctive

\begin{tabular}{|c|c|c|c|c|}
\hline Variable & & Group $1(F C L T)(n=25)$ & Group $2(F C D T)(n=25)$ & $p$-value \\
\hline \multirow[t]{2}{*}{ Age } & Range & 40-70 years & $42-80$ years & 0.634 \\
\hline & Mean \pm SD & $55.28 \pm 8.87$ & $56.60 \pm 10.51$ & \\
\hline \multirow[t]{2}{*}{ Sex } & Male & $14(56 \%)$ & $17(68 \%)$ & 0.382 \\
\hline & Female & $11(44 \%)$ & $8(32 \%)$ & \\
\hline \multirow[t]{2}{*}{ Eye } & Left & $13(52 \%)$ & $12(48 \%)$ & 0.77 \\
\hline & Right & 12 (48\%) & 13 (52\%) & \\
\hline
\end{tabular}

p-value $\geq 0.05$ nonsignificant

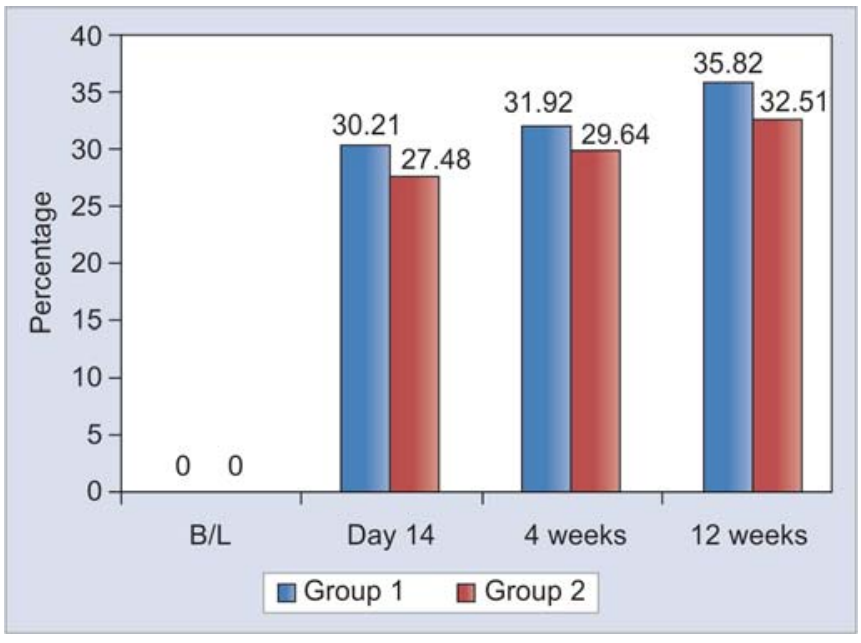

Graph 1: Percentage reduction in IOP of the two groups at $9 \mathrm{AM}$

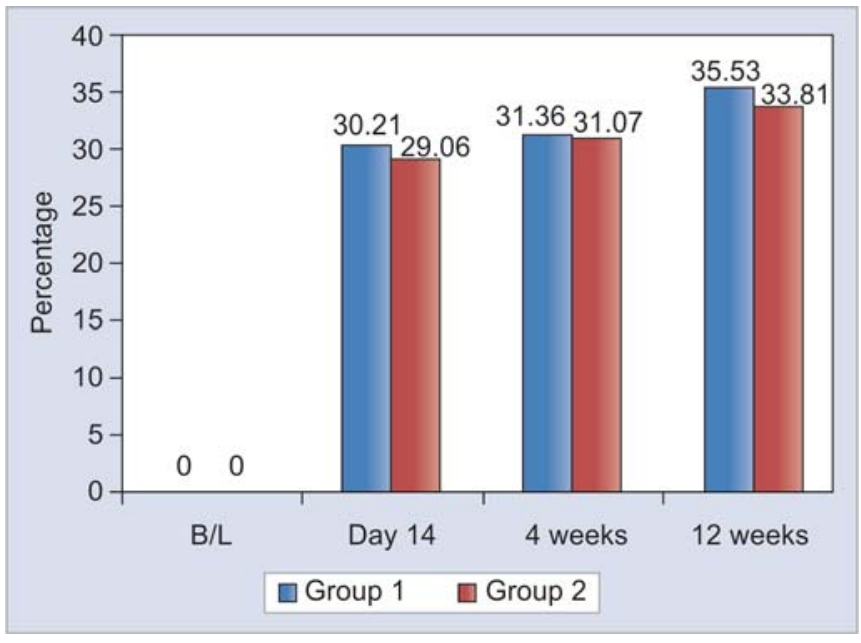

Graph 2: Percentage reduction in IOP of the two groups at 12 noon 


\begin{tabular}{llcccccc}
\hline \multicolumn{7}{c}{ Table 2: IOP after 12 weeks for both time durations } \\
\hline Time & Interval & Group 1 (mean \pm SD) & Group 2 (mean \pm SD) & $t$-value & $p$-value & Sig. \\
\hline \multirow{2}{*}{9 AM } & B/L & $27.88 \pm 2.186$ & $27.56 \pm 2.103$ & - & - & - & - \\
\multirow{2}{*}{12 noon } & 12 weeks & $17.92 \pm 0.86$ & $18.52 \pm 0.770$ & -2.595 & -013 & - & - \\
& $24-35$ & $27.72 \pm 2.301$ & $27.92 \pm 2.431$ & - & -3.292 & 0.002 & $\mathrm{~S}$ \\
\hline
\end{tabular}

$p<0.05$ is significant; Significant difference in reduction of IOP favoring group 1 only at 12 weeks interval

Table 3: Adverse effect of study drugs

\begin{tabular}{lcc}
\hline Ocular adverse effects & Group A & Group B \\
\hline Conjunctival hyperemia & 4 & 1 \\
Burning/stinging sensation & 1 & 2 \\
Foreign body sensation & 1 & 1 \\
Photophobia & - & 1 \\
Eye pain & - & 4 \\
\hline
\end{tabular}

therapy may be required. To improve compliance and convenience to the patient two drugs can be given in a fixed combination of the two individual components.

In this 12 weeks study, the reduction in IOP brought about by the drugs in both groups 1 and 2 was statistically significant at all time intervals. The difference in final IOP achieved in the two groups was not statistically significant for 2 and 4 weeks periods but at week 12 the latanoprost/ timolol combination showed more efficacy. The decrease in IOP from baseline to 12 weeks in group 1 was 9.960 and 9.880 at $9 \mathrm{AM}$ and 12 noon respectively. In group 2, this decrease was 9.040 and 9.400. The difference in IOP reduction between two groups was statistically significant at 12 weeks for both time readings $(\mathrm{p}=0.013$ and 0.002 respectively.

In a study done by Miglior et al ${ }^{14,15}$ in 2009, compared the efficacy of FC latanoprost/timolol and FC dorzolamidetimolol. It was found that mean reductions in daytime IOP from baseline to week 12 was by $-9.7 \mathrm{~mm} \mathrm{Hg}$ for FC latanoprost-timolol and $-9.5 \mathrm{~mm} \mathrm{Hg}$ for FC dorzolamide/ timolol, indicating that neither FC is inferior to the other.

The results of this study parallels the result of a 3-month, randomized, parallel-group, evaluator-masked study, ${ }^{16}$ in 253 subjects, in which it was found that FC latanoprost/ timolol reduced the mean daytime IOP by $1 \mathrm{~mm}$ Hg more than the comparator $(p=0.005)$ and the mean IOP levels after 3 months of treatment were significantly lower in the FC latanoprost/timolol group at 0800 and 1600 hours ( $\mathrm{p}<0.01$ for both comparisons).

Cvenkel et a ${ }^{17}$ did a similar study in 2008 and found that patients treated with dorzolamide/timolol FC and latanoprost/timolol FC have no statistically similar ocular hypotensive effect.

Both FC agents were well tolerated. In all, six out of 25 (25.9\%) subjects in the FC latanoprost/timolol group and seven out of 25 (30.4\%) in the FC dorzolamide/timolol group reported adverse events. The most frequently occurring adverse events related to study medications were eye pain, eye irritation, conjunctival hyperemia, and taste disturbance, which were not long lasting. The most commonly noted ocular adverse events in the FC latanoprost/timolol group were eye pruritus and conjunctival hyperemia; most frequently reported ocular adverse events in the FC dorzolamide/timolol group were eye pain, blurred vision, stinging, dry eyes. In general, both fixed-combination drugs were well tolerated. Since, one drug was given once a day and the other twice a day this study could not be blinded which was a limitation of this study.

\section{CONCLUSION}

From our study, it can be concluded that IOP lowering efficacy of latanoprost/timolol is superior to dorzolamide/ timolol.

In general both the fixed combinations were well tolerated. There was no significant differences in drug related ocular or systemic side effects.

However, given the small differences in IOP lowering between the two therapies, assessing the effects of differences in dosing and tolerability on patient compliance and patient outcomes should be the goals of further research.

\section{REFERENCES}

1. Van Buskirk EM, Cioffi GA. Glaucomatous optic neuropathy. Am J Ophthalmol 1992;113(4):447-52.

2. Quigley HA. Number of people with glaucoma worldwide. Br J Ophthalmol 1996;80:389-93.

3. Resnikoff S, et al. Global data on visual impairment in the year 2002. Bull World Health Organization 2004;82:844-51.

4. Quigley HA. Glaucoma. Lancet 2011 Apr 16;377(9774):1367-77.

5. George R, Ve RS, Vijaya L. Glaucoma in India: Estimated burden of disease. J Glaucoma 2010 Aug;19(6):391-97.

6. Herschler J, Litin BS. Biochemical abnormalities in the aqueous in chronic open angle glaucoma. Ophthalmic Surg 1987 Nov; 18(11):792-95.

7. Weinreb RN. A rationale for lowering intraocular pressure in Glaucoma. Surv Ophthalmol 2001;45(4):S335-36.

8. Hoyng PF, Van Beek LM. Pharmacological therapy for Glaucoma. Drugs 2000 Mar;59(3):411-34.

9. Hommer A. Role of fixed combinations in the management of open-angle glaucoma. Expert Rev Pharmacoecon Outcomes Res 2011 Feb;11(1):91-99.

10. Neymark N, Buchholz P, Honrubia F, Kobelt G. The costs of treating glaucoma with combinations of topical drugs in Spain. Eur J Ophthalmol 2008 Jan-Feb;18(1):52-59. 
11. Higginbotham EJ, Feldman R, Stiles M, Dubiner H. Latanoprost and timolol combination therapy vs monotherapy: 1-year randomized trial. Arch Ophthalmol 2002;120(7):915-22.

12. Schwenn O, Heckmann B, Guzy C, Miller PJ. Long-term effect of latanoprost/timolol fixed combination in patients with glaucoma or ocular hypertension: A prospective, observational, noninterventional study. BMC Ophthalmol 2010 Sep 8;10:21.

13. Bell NP, Ramos JL, Feldman RM. Safety, tolerability and efficacy of fixed combination therapy with dorzolamide hydrochloride $2 \%$ and timolol maleate $0.5 \%$ in glaucoma and ocular hypertension. Clin Ophthalmol 2010 Nov 22;4:1331-46.

14. Miglior S, Grunden JW, Kwok K. Efficacy and safety of fixed combinations of latanoprost/timolol and dorzolamide/timolol in open-angle glaucoma or ocular hypertension. Eye 2010;24: 1234-42.

15. Resnikoff S, Pascolini D, Etya’ale D, Kocur I, Pararajasegaram R, Pokharel G, et al. Global data on visual impairment in the year 2002. Bull World Health Organ 2004;82: 844-51.

16. Shin DH, Feldman RM, Sheu WP. Efficacy and safety of fixed combinations latanoprost/timolol versus dorzolamide/timolol in patients with elevated intraocular pressure. Ophthalmology 2004 Feb;111(2):276-82.
17. Cvenkel B, Stewart JA, Nelson LA, Stewart WC. Dorzolamide/ timolol fixed combination versus latanoprost/timolol fixed combination in patients with primary open-angle glaucoma or ocular hypertension. Curr Eye Res 2008 Feb;33(2):163-68.

\section{ABOUT THE AUTHORS}

\section{Sukhsagar Ratol (Corresponding Author)}

Postgraduate Student (Final Year), Department of Pharmacology, MM Institute of Medical Sciences and Research, Mullana, Ambala Haryana, India, e-mail: docsukhsagar@yahoo.com

\section{Rani Walia}

Professor and Head, Department of Pharmacology, MM Institute of Medical Sciences and Research, Mullana, Ambala, Haryana, India

\section{Mridu Chaudhry}

Associate Professor, Department of Ophthalmology, BPS Govt. Medical College for Women, Khanpur Kalan, Sonepat, Haryana, India 